\title{
AVATARES DE LA PSICOLOGÍA CLÍNICA, ESPECIALIDAD SANITARIA DE LA PSICOLOGÍA. INFORME DE ACTIVIDAD DE LA COMISIÓN NACIONAL (CNEPC) JUNIO 2003/JUNIO 2004
}

\author{
BEGOÑA OLABARRÍA \\ Presidenta de la Comisión Nacional de la Especialidad de Psicología Clínica
}

(Aceptado en julio de 2004)

Decía en las Jornadas del presente año de la AEPCP celebradas en Sevilla, que creía no equivocarme al decir que en el 2002 se cierra la etapa de la institucionalización de la Especialidad en nuestro país: Por una parte, se dictaron las favorables sentencias del Tribunal Supremo en torno a la Psicología Clínica como especialidad sanitaria de la Psicología frente a los Recursos Contencioso-Administrativos interpuestos contra su existencia.

Por otra también en el mismo año se alcanzaba la publicación en el BOE de la Orden Ministerial PRE/1107/2002, por la que se regulan las vías transitorias de acceso al título de Psicólogo Especialista en Psicología Clínica en desarrollo de lo dispuesto en el Real Decreto 2490/98, abriendo así el proceso para hacer operativa la especialidad.

Y decía también que se iniciaba otro momento distinto en que podían hacerse patentes las diferencias (algunas sustanciales) que hasta cierto punto se habían ido manteniendo más o menos tapadas en tanto se venía trabajando por la construcción de la especialidad y su modelo de formación en el Sistema Nacional de Salud.

Se abría pues una etapa en donde el esfuerzo a mi juicio había de centrarse en la puesta en valor de la especialidad, desde el sostenido convencimiento de que la Psicología Clínica es portadora de un saber que puede y debe contribuir a una atención integral biopsicosocial de los ciudadanos en su contexto comunita- rio y en los tres niveles de prevención. $Y$ que a ello, a su realización -en niveles y cotas bajos actualmente- debería la CNEPC dedicar su esfuerzo.

La Comisión Nacional ha desarrollado trabajos vinculados a lo que pretendimos nueva fase y que entendimos debía dirigirse hacia la normalización de la especialidad en el Sistema Nacional de Salud desde esa perspectiva de puesta en valor de la misma.

Los trabajos desarrollados en el último año por la Comisión Nacional han estado dirigidos a:

1. Valorar e Informar cada uno de los expedientes de solicitud de homologación de título de especialista trasladados a tal fin por el Ministerio de Educación y todo ello siguiendo las disposiciones transitorias definidas en el Real Decreto 2490 y de acuerdo con lo establecido en la Orden Ministerial antes reseñada.

El ingente trabajo que ello supone se está realizando sobre la previa definición por los miembros de la Comisión Nacional de criterios de valoración para cada una de las cuatro disposiciones transitorias definidas en la Orden Ministerial y cuya aplicación se realiza en la rigurosa revisión de cada uno de los expedientes de solicitud.

Hasta el momento, de los 10.500 expedientes presentados se han valorado e informado alrededor de 2200 expedientes. 
La CNEPC ha revisado el procedimiento para ajustar los criterios específicos correspondientes a cada una de las Disposiciones Transitorias buscando en todo momento que sea trasparente, equitativo, objetivo y justo. En cualquier caso cabe destacar que en todas y cada una de las revisiones y decisiones la Comisión ha buscado el consenso y ha alcanzado unánime acuerdo.

En el mes de octubre de 2003 la Comisión recibió la visita de D. Marc Soler, entonces Subdirector General de Recursos Humanos del Ministerio de Sanidad y de D. ${ }^{\mathrm{a}}$ Emilia Sánchez Chamorro, Subdirectora de Especialidades en Ciencias de la Salud del Ministerio de Educación, quienes expresaron su agradecimiento por la diligencia y el rigor con el que se lleva a cabo todo el proceso. Informaron de la recepción en ambos ministerios de numerosos escritos de psicólogos que están trabajando en el SNS, solicitando la mayor celeridad posible en la revisión de sus expedientes, dado que estaban pendientes de la reclasificación de sus plazas para su conversión en plazas de facultativos especialistas en el SNS, y esperando presentarse a oposiciones, principalmente la OPE de INSALUD.

Esta situación de reclasificación como especialistas se deriva de la aplicación de la Disposición Adicional segunda del Real Decreto 2490 por el que se crea y regula la Especialidad y es relativa al personal estatutario, es decir, psicólogos que trabajan en el Sistema Nacional de Salud; Disposición Adicional cuya aplicación se ha iniciado en algunas Comunidades Autónomas, que a su vez se han dirigido a la Admón. Central interesándose en esta materia. Por todo ello, ambos Subdirectores Generales iniciaron la búsqueda de procedimientos que facilitasen abordar soluciones en esta materia y al tiempo aumentasen la eficacia de los trabajos de homologación. Para ello propusieron reorganizar las revisiones de expedientes y dar traslado de los mismos a la CNEPC por Disposiciones Transitorias, empezando por la Disposición Transitoria Segunda, que se refiere a los profesionales que trabajan en servicios públicos realizando labores clinico/asistenciales.

La propuesta fue valorada positivamente por todos los miembros de la Comisión Nacional y la experiencia desarrollada demuestra que la eficacia es mayor: vemos con mayor precisión y celeridad los expedientes cuando en la mayoría de ellos aplicamos los criterios que corresponden a una de las Disposiciones Transitorias y no llegan con una agrupación de mezcla de todos los grupos (es preciso señalar que, no obstante, en cada una de las reuniones se revisan expedientes de otras Disposiciones Transitorias, si bien mayoritariamente los enviados en los últimos seis meses corresponden a la Segunda, como ha quedado dicho).

Así mismo y como consta en el acta de 31 de octubre D. Mariano Vera, Secretario General del COP, expresó en visita a la Comisión Nacional acompañado por ambos subdirectores generales, en su nombre y en el del COP, el agradecimiento a los miembros de la Comisión por el trabajo enorme que ésta está realizando y el empeño puesto por todos sus miembros, así como por parte de ambos ministerios, en resolver rigurosa y satisfactoriamente el proceso y cuanto antes. Así mismo expresó que había dado traslado a ambos Ministerios de la satisfacción del COP por el modo en que se está realizando el, en palabras del mismo, «ingente» trabajo.

Los miembros de la CNEPC dieron traslado a ambas subdirecciones generales de los temores de algunos profesionales que están trabajando en instituciones sanitarias de la red pública por la posibilidad de que algunos no obtuvieran el título de especialista y que pudieran así verse de distintas maneras perjudicados, 
y más tras la promulgación de la Ley de Ordenación de las Profesiones Sanitarias (LOPS). La respuesta obtenida fue que los derechos adquiridos como personal estatutario o como funcionario son siempre respetados, lo que significa que no disponer del título de Especialista en Psicología Clínica no quiere decir que se deje de ser funcionario en el mismo nivel que se ocupara antes.

Priorizar la revisión de los expedientes que corresponden a la Disposición Transitoria Segunda (instituciones públicas) se propuso por parte de la Administración, como ha quedado dicho, para agilizar el proceso de revisión de expedientes y fue aceptada por todos los miembros de la CNEPC y en plena sintonía.

Los criterios por los que la CNEPC aceptó la propuesta constan en las actas de la CNEPC y son en resumen éstos:

a) El número menor de requisitos a revisar (menor variabilidad de los expedientes, en comparación con los de las Disposiciones Transitorias Tercera y Cuarta), lo que hacía más fácil la revisión a los funcionarios del Ministerio de Educación, que son los que deben hacer el primer filtrado para constatar que el solicitante reúne requisitos básicos de acceso (por ej. la titulación) y ha remitido todos los documentos exigibles, y de no ser así, solicitárselos; y también a los del M. ${ }^{\ominus}$ de Sanidad, quienes, tras la valoración de la CNEPC, revisan de nuevo el expediente a la luz del informe emitido.

b) El número de solicitantes por esta Disposición Transitoria Segunda.

c) El hecho de que, de facto, mayoritariamente estos expedientes fueron remitidos antes que los de la Disposición Transitoria Tercera y la Cuarta, y por tanto, su orden de llegada era anterior. d) Facilitar el trabajo de revisión de los expedientes a la CNEPC: no se es igual de eficaz si se revisan requisitos diferentes cada vez, que si se hace según bloques o agrupaciones de perfiles y/o requisitos.

e) Contribuir a dotar del estatus que corresponde a la especialidad de Psicología Clínica en el Sistema Nacional de Salud, sus cometidos y tareas, a través de la facilitación de los tiempos para la reclasificación de sus profesionales. Ello ha de contribuir a desarrollar los cometidos de la especialidad con base adecuada.

He de destacar que el objetivo es que todos los expedientes que lleguen en cada reunión a la CNEPC, se examinen el mismo día de su llegada: un promedio de 130-150 expedientes en maratonianas sesiones de trabajo (reuniones que siempre cuentan en el Orden del Día con la revisión de expedientes de solicitud de homologación y también con otros asuntos relevantes referidos a la especialidad), pues la tarea de revisión de todos y cada uno de los expedientes de solicitud de convalidación se realiza con la máxima atención.

El procedimiento adoptado ha buscado garantizar la legitimidad de las actuaciones de la CNEPC, que ha logrado alcanzar tanto global como específicamente en estos trabajos expreso reconocimiento por cuantos han participado del proceso de homologación desde la propia Administración y cuantos han tenido ocasión de conocerlas desde distintos ámbitos, y del que han destacado la equidad, el rigor, la claridad y transparencia de las decisiones y actuaciones realizadas.

Por otra parte tal vez resulte de interés plantear algunos extremos sobre el proceso de homologación que corresponde a la Disposición Transitoria Tercera (ejercicio privado de la profesión). Concreta- 
mente me referiré a las expectativas -a veces presentes en compañeros que han solicitado la homologación del título de especialista por dicha Disposición- de alcanzar automáticamente el título una vez que han obtenido y presentado en su expediente el informe del COP acreditando su ejercicio como clínico. Estos informes que, previo pago por los interesados, ha realizado el COP para quienes se los solicitaron para poder optar a la expedición del título de especialista son preceptivos según la Orden Ministerial PRE/1107/2002, para todos los que solicitaban la homologación del título por esta Disposición Transitoria Tercera.

Se trata de un requisito imprescindible, pero no único.

Así quedó establecido desde los primeros trabajos para la definición de criterios y procedimientos en la CNEPC en la valoración de expedientes de solicitud de homologación del título de especialista de acuerdo al R.D. 2490/98 y la O.M. PRE/1107/2002.

Por ello, los informes colegiales están incorporados al expediente de solicitud de homologación y son vistos siempre expresamente en la valoración que realiza la CNEPC. A continuación se valoran el resto de los requisitos previstos legalmente. Seguramente ello tiene su lógica propia, además de la legal: Dichos informes colegiales consisten en un informe estándar, en el que constan 2 elementos: los años que ha estado colegiado el solicitante y la expresión del COP de que ha ejercido como psicólogo clínico. No figuran -y no se ha dado traslado a la CNEPC - cuáles han sido los procedimientos y criterios para definir el ejercicio profesional de un psicólogo como de especialista en Psicología Clínica.

De hecho lo que hemos visto hasta ahora en la CNEPC es la alta variabilidad de ejercicio presentada en estos expedientes de homologación por la Disposición Transitoria Tercera y que incorporan precepti- vamente el mismo Informe colegial: junto a profesionales que presentan clara formación y ejercicio clínicos, existen otros que contando con la misma certificación colegial, presentan como ejercicio, por ej., ser propietarios de una guardería infantil.

Hasta ahora, la CNEPC asume (R.D.2490/98 y O.M. PRE/1107/2002) la ingente tarea de valorar e informar todos los expedientes presentados desde el $\mathrm{M}^{2}{ }^{2}$ Educación y ello responsable y plenamente: revisa cada uno de los expedientes de cada una de las Disposiciones Transitorias atendiendo a todos los requisitos. En la Disposición Transitoria Tercera, verifica la existencia del informe preceptivo del COP y a continuación verifica y valora el resto de los requisitos (ejercicio y formación) de cada solicitante y las fechas de los mismos.

2. Conviene alertar acerca de la próxima publicación de un nuevo Real Decreto (Proyecto de R.D. por el que se modifican las disposiciones transitorias del Real Decreto 2490/1998, por el que se crea y regula el Título Oficial de Psicólogo Especialista en Psicología Clínica).

Lo fundamental de este próximo Real Decreto es que modificará, abriéndolos, las fechas de valoración del ejercicio profesional, el acceso a la solicitud a los profesores de Escuelas Universitarias y los plazos de presentación de nuevas solicitudes de homologación.

Este Proyecto, fue remitido por la Secretaría General Técnica del M. ${ }^{\otimes}$ Sanidad, se discutió ampliamente y quedó valorado positivamente en función de la necesidad de corregir los daños que la dilación habida entre la publicación del Real Decreto 2490/98 de creación de la especialidad (1998) y la publicación de la Orden Ministerial PRE/1107 (2002) provocaban a muchos profesionales en ejercicio. Se acuerda, en febrero de 2004, dar informe favorable a la Administración respecto de su publicación, que se encuen- 
tra en este momento pendiente y que posiblemente será próxima.

Es evidente por tanto que el número de solicitudes de homologación del título de especialista en Psicología Clínica aumentará tras la publicación de este Real Decreto de ampliación lo que sin duda aumentará los tiempos de valoración y resolución.

3. Incremento de plazas PIR.

La CNEPC viene reclamando año a año un significativo aumento de plazas PIR, como figura en las actas de la CNEPC, sin haber obtenido una respuesta satisfactoria. La AEPCP ha respaldado expresamente estas solicitudes de aumento de plazas PIR.

El número de plazas PIR deviene de una toma de decisión compleja que incluye, además del marco ministerial, el de las CCAA y las gerencias de Área (por cuanto son éstas quienes han de dotar las plazas económicamente).

Al tiempo, debe resaltarse que las plazas PIR han de estar directamente relacionadas con las plazas de psicólogos clínicos en los equipos de las Unidades Docentes Acreditadas del SNS y que estas plazas no han tenido el necesario incremento que desde luego la formación de especialistas requiere, como la demanda de atención en los servicios por parte de los ciudadanos, aconseja.

No obstante lo anterior, cabe señalar el incremento de 1 plaza más en Toledo, 1 en Valladolid y 2 en Madrid. Así mismo se ha aprobado nueva acreditación docente para la formación especializada en Psicología Clínica: la Unidad del Complejo Hospitalario de Ciudad Real.

\section{Análisis y discusión del desarrollo normativo}

- Ley de Autonomía del paciente. La CNEPC no fue consultada y conoció el texto de la Ley tras su publicación.
No obstante ha debatido y expresado en acta su disentimiento por la expresión del término "médico» y no "facultativo» en alguno de sus contenidos, lo que podría confundir la responsabilidad en el ejercicio responsable de la especialidad.

- Ley de cohesión y Calidad del SNS.

La CNEPC no fue consultada y conoció el texto tras su publicación. No obstante y por vías ajenas a la Admón. Conoció alguno de sus borradores, por lo que desde febrero hasta la aprobación de la Ley en mayo de 2003 ha sido objeto de un análisis pormenorizado en la CNEPC por su reconocida trascendencia.

Se ocupa entre otros ámbitos de las prestaciones en Salud Pública, Atención Primaria, Especializada, dentro de ella Salud Mental y urgencias, así como de la definición de la Cartera de Servicios del Sistema Nacional de Salud.

Tiene especial importancia en el capítulo de la planificación y formación de profesionales la creación de la Comisión de Recursos Humanos. Dicha Comisión tendrá, entre otras funciones, la de supervisar los programas de formación de especialistas, propuestos por las Comisiones Nacionales específicas, así como la de supervisar el número de profesionales necesarios en cada convocatoria. En ella participan, entre otros organismos, las Comisiones Nacionales de diferentes Especialidades Sanitarias, a excepción de la Comisión Nacional de Psicología Clínica.La CNEPC solicitó su incorporación y participación en la Comisión de Recursos Humanos, y, entre tanto se creara, reiteró la petición de participación en el vigente Consejo Nacional de Especialidades Médicas y el cambio de denominación para permitir la incorporación de todas las Especialidades Sanitarias. En diciembre de 2003 el Consejo In- 
terterritorial dio el visto bueno al Proyecto de Decreto presentado por el Ministerio de Sanidad, por el que se determina la composición y desarrollo de la Comisión de Recursos Humanos del Sistema Nacional de Salud.

Por ultimo la CNEPC decidió solicitar de nuevo la incorporación en todas las estructuras docentes ministeriales y la participación en todos los temas vinculados a la formación de especialistas, formación continuada, y planificación de recursos humanos.

- Real Decreto 1277 por el que se establecen las bases generales sobre autorización de centros, servicios y establecimientos sanitarios, aprobado en octubre 2003.

Al finalizar 2003 la CNEPC discutió y analizó este $\mathrm{RD}$, que no había conocido con anterioridad y sobre el que no fue consultada durante su elaboración, y que es un desarrollo de la Ley de Cohesión y Calidad del SNS.

Expresamente en su Introducción el $R D$ introduce la idea de que no entra en la definición de perfiles profesionales, sino en los requisitos para conceder autorización para la apertura de centros, servicios y establecimientos sanitarios. Pero la cerrada concepción de los requisitos, fue valorada negativamente por la CNEPC.

Se acordó por unanimidad expresar desacuerdo por el marcado carácter corporativista medicalizado de la atención sanitaria y realizar propuesta expresa de modificación de aquellos contenidos especialmente lesivos para el ejercicio de la Psicología y la Especialidad de Psicología Clínica.

Por ello se propuso al Ministerio la modificación los contenidos de algunas unidades y/o programas y, de conformidad con el Real Decreto 2490/98 por el que se crea y regula la Especialidad de Psicología Clínica, y su susti- tución por lo que se expresa a continuación:

C.2.5.10 Centros de reconocimiento médico-psicológico: centros sanitarios donde se efectúan las revisiones medico-psicológicas e informes.............

C.2.5.11 Centros de Salud Mental: centros sanitarios en los que se realiza el diagnóstico y tratamiento en régimen ambulatorio de los trastornos y las enfermedades mentales.......

U.36 Tratamiento del dolor: unidad asistencial en la que un especialista sanitario es responsable de aplicar.......

U.65 Hospital de día: unidad asistencial donde, bajo la supervisión o indicación de un especialista sanitario se lleve a cabo..........métodos de tratamiento que requieran durante unas horas atención continuada sanitaria pero no el internamiento en el hospital.

U.70 Psicología Clínica: unidad asistencial el la que un psicólogo especialista en Psicología Clínica es responsable de realizar diagnósticos, evaluaciones y tratamientos de carácter psicológico de los trastornos mentales y de aquellos fenómenos psicológicos, conductuales y relacionales que inciden en el proceso salud/enfermedad de los seres humanos y en su calidad de vida.

- Ley de Ordenación de las Profesiones Sanitarias (LOPS).

Esta Ley ha sido objeto de análisis y discusión pormenorizada desde enero de 2003 en que la $\mathrm{CN}$ tuvo ocasión de conocer uno de sus borradores por fuentes ajenas a las de la Administración, dado que la CNEPC no ha sido consultada. 
Sin embargo esta Ley, que viene a ser complementaria de la Ley de Cohesión, fue debatida en la CNEPC al considerarla de enorme trascendencia por cuanto regula los aspectos básicos de las profesiones sanitarias, la estructura general de la formación de los profesionales, su desarrollo profesional en el SNS y su participación en la planificación y ordenación de las profesiones sanitarias.

Mediante esta Ley la Administración Pública se hace garante de las prestaciones sanitarias a la población y de su calidad.

Se señala entre otras características el cambio de denominación del Consejo Nacional de Especialidades Médicas por el de Consejo Nacional de Especialidades en Ciencias de la Salud, hecho que posibilitaría la incorporación de la CNEPC al mismo, cuestión ésta repetidamente solicitada por la CNEPC.

En julio y a raíz de la solicitud de la CNEPC y de las múltiples gestiones realizadas por las asociaciones y organizaciones representadas en la Comisión, entre ellas la AEN, tanto ante los ministerios como ante los grupos parlamentarios, el Ministerio de Sanidad da respuesta parcialmente positiva a solicitudes de modificación hechas: la incorporación de los psicólogos clínicos entre los facultativos sanitarios. Este cambio abre la puerta para a la inclusión en el controvertido Estatuto Marco del Personal Sanitario de los Servicios de Salud.

No obstante lo anterior, la CN había expresado su interés en torno a la incorporación de la licenciatura en Psicología entre las profesiones sanitarias en el entendimiento de que, si bien no se trataba de un asunto de competencia de la CNEPC, aportaba con ello una opinión con la que contribuir al imprescindible debate para el establecimiento de acuerdos en la comunidad científico-profesional de la Psicología en su conjunto por las implicaciones teórico-técnicas, de for- mación en los planes de estudio, deontológicas, en los derechos de usuarios, etc., que este aspecto implicaba en el conjunto de una licenciatura y profesión polivalente en sus campos de intervención. Cuestiones que, como ya ha quedado dicho, salían del ámbito competencial de la CNEPC para entrar abiertamente y en primer lugar en el de las facultades de Psicología y en el M. ${ }^{\circ}$ Educación, cuyos responsables, cometidos y competencias son y siempre han sido muy respetados por la CNEPC.

Por otra parte, también se hicieron propuestas para la incorporación de cambios en la composición de la Comisión Consultiva Profesional y la formación continuada, los cuales tampoco fueron atendidos.

La Ley quedó aprobada y publicada en noviembre de 2003.

Tras su publicación, la alarma suscitada entre muchos colegas al entender que la no inclusión de la licenciatura en Psicología entre las profesiones sanitarias descritas en la LOPS, implicaba de facto la prohibición de ejercer en el campo de la salud con la licenciatura, llevó a la CNEPC a generar diversas consultas en Ministerio de Educación y Ministerio de Sanidad, recibiendo en diferentes ocasiones repetidas informaciones en sentido contrario: La LOPS describiría las profesiones sanitarias, pero no entraría en prohibir un ejercicio profesional en el campo de la salud a los licenciados en Psicología por cuanto el título de licenciado habilita para la intervención profesional en cuantos campos de intervención lo resulten para la Psicología, entre ellos el de la salud, y siempre y cuando no se arrogasen los licenciados no especialistas en Psicología Clínica, dicha denominación ante requerimientos de atención especializada.

Esta información ha sido ratificada por la asesoría jurídica ministerial y traslada a la CNEPC en su reunión del pasado día 
14 de mayo, tras el resultado electoral y los consiguientes cambios habidos en el Gobierno, como consta oportunamente en el acta de la misma.

La CNEPC ha recibido los escritos enviados por la AEPCP sobre ésta y otras cuestiones (ver posiciones de la AEPCP actualmente presentadas en la web de la Asociación y en la Revista) como aportaciones específicas para los debates habidos y posiciones sostenidas en y por la CN.

- Catálogo de Técnicas y Procedimientos del Sistema de Información Cartera de Servicios (SICAR).

En octubre de 2003 la CNEPC accede (casualmente y fuera de las fuentes ministeriales) y discute este Catálogo, publicado por el Ministerio de Sanidad en el 2002. Elaborado por el INSALUD queda presentada la edición como borrador de una futura de Cartera de Servicios del SNS.

La Cartera de Servicios es un compromiso que instaura la LOPS y que la Administración Sanitaria manifiesta recoger, teniendo como primer objetivo su definición.

El texto Catálogo de Técnicas y Procedimientos del Sistema de Información Cartera de Servicios (SICAR) expone las prestaciones del SNS por especialidades y excluye cualquier mención de las prestaciones correspondientes a la Especialidad de Psicología Clínica, por lo que se acuerda en la CNEPC reclamar la presencia de la Psicología Clínica en los nuevos y sucesivos proyectos de Cartera de Servicios (realizándose gestiones específicas al respecto), entendiendo que la no inclusión constituye un daño para la especialidad, para su formación y para el Sistema Nacional de Salud.

5. Revisión y elaboración de documentación. Se ha revisado y reelaborado la solicitud de acreditación para la for- mación especializada en Psicología Clínica, así como la totalidad de sus anexos:

- Características que deben reunir los servicios sanitarios para quedar acreditados como Unidades Docentes Acreditadas (UDAs).

- Fuentes documentales y bibliográficas organizadas por bloques temáticos.

- Relación de pruebas básicas para el psicodiagnóstico y evaluación psicológica, contemplando nuevas áreas de exploración tanto en adultos como en infancia y adolescencia.

En esta misma línea y haciendo referencia a documentos elaborados con anterioridad la Comisión expresa su preocupación, manifestada reiteradamente por todos los miembros, por la paralización de la puesta en marcha del nuevo Libro del Residente. El Libro del Residente es instrumento fundamental para el seguimiento individualizado de la formación de los especialistas de todas las especialidades (Orden Ministerial de 22 de junio de 1995). El existente en la actualidad se encuentra en completo desuso por tratarse de un Libro común a todas las especialidades y completamente inadecuado para la especialidad de Psicología Clínica, entre otras. Por esta razón y de mutuo acuerdo, las Comisiones Nacionales de Psicología Clínica y Psiquiatría hace dos años aproximadamente, diseñaron un nuevo Libro del Residente de manera diferenciada del resto de especialidades y discriminada entre ambas, Libro que quedó elaborado y aprobado por ambas Comisiones Nacionales después de haber sido contrastado en 12 Unidades Docentes acreditadas de todo el Estado. Se acordó reiterar la solicitud a los Ministerios para su inmediata puesta en marcha, que todavía esperamos. 
6. Mención especial merece la elaboración del nuevo Programa de la Especialidad de Psicología Clínica, que centró una parte importante de los esfuerzos de la Comisión Nacional durante más de dos años, con estudios, debates y consultas hasta la elaboración del Borrador definitivo, y que quedó aprobado por unanimidad de sus miembros en marzo del pasado año. Se encuentra aún pendiente de la valoración de los ministerios implicados: Sanidad y Educación.

De él mencionar al menos y brevemente alguno de los aspectos tal vez más relevantes, como la solicitud de ampliación de un año más de duración para el Programa formativo y la incorporación de nuevos programas y ámbitos de formación, sistema de rotaciones, áreas de especial interés como Psicología Clínica de niños y adolescentes, Programas de interconsulta y apoyo e intervención en y con distintos servicios de Hospital General, y otros.

Esperamos que esta legislatura vea aprobado el nuevo Programa de Formación que mejora sustancialmente diversos aspectos del vigente, que, aprobado en 1995 y publicado en 1996, dos años antes de que viera la luz el RD 2490/98 por el que se crea y regula la especialidad, se vió constreñido por la situación de transitoriedad y falta de oficialización de la especialidad.

El Proyecto de Programa cuenta con el explícito respaldo de la AEPCP ante la Administración y continúa a la espera de su valoración por la Administración.

7. Memoria anual de la formación Especializada en Psicología Clínica (PIR).

En enero de 2004 la CNEPC elabora una Guía para la elaboración de una memoria anual, que permita hacer un seguimiento de la formación PIR en los servicios sanitarios que configuran cada Unidad Docente Acreditada (UDA) para la formación de especialistas en Psicología Clínica.
Se acuerda proponer a la Administración su envío a todas las Unidades Docentes Acreditadas para que, de acuerdo con la Orden de 22 de junio de 1995 por la que se regulan las Comisiones de Docencia y los sistemas de evaluación de las especialidades, permita el seguimiento adecuado del programa formativo.

8. En aplicación del acuerdo de la CNEPC de realización de auditorias anuales sobre UDAs como instrumento de verificación del grado cumplimiento del Programa de Formación de Especialistas, en 2003 se solicita auditoría sobre la UDA del Hospital Ramón y Cajal de Madrid.

Del exhaustivo Informe del servicio de auditoría, que muestra una vez más, una calidad excelente, se desprenden importantes incumplimientos del Programa de Formación desarrollado en esta UDA.

Por ello, la CNEPC acuerda solicitar modificaciones de acuerdo a unas recomendaciones que traslada a la Administración, solicitando sean remitidas a los responsables de la UDA, y sobre las que compromete seguimiento.

9. El 5 de marzo del presente 2004, D. Manuel Berdullas, representante del COP en la CNEPC, al inicio de la reunión presenta una copia de la carta que D. Francisco Santolaya, Decano del COP, dirige al Director General de Recursos Humanos del Ministerio de Sanidad y se retira de la CNEPC.

En dicha carta el decano del COP requiere a las autoridades ministeriales la inmediata renovación de los miembros de la CNEPC, aduciendo que se han cumplido los tiempos establecidos y comunica la retirada de su representante en la CNEPC en tanto no se hayan producido dichos cambios arguyendo pérdida de confianza en el funcionamiento de la Comisión, que parece justificar por la decisión del Ministerio de resolver con prioridad los expedientes correspondien- 
tes a la Disposición Transitoria Segunda (Servicios Públicos).

Así mismo vierte descalificaciones sobre la presidenta de la Comisión.

En torno a la renovación de la CNEPC alguna aclaración: La renovación de los miembros que componen las Comisiones Nacionales (CNs) de Especialidades compete a la Administración, y no a los que forman parte de las mismas: Esta renovación debe normativamente producirse, como en todas las CNs de todas las especialidades, a los cuatro años del inicio de las actuaciones de la Comisión de que se trate. Tal fecha corresponde por tanto al pasado noviembre 2003 en lo que se refiere a la CNEPC.

Conviene decir que la práctica de la Admón. en esta materia muestra que las renovaciones en las Comisiones Nacionales habitualmente se desarrollan con lentitud, como es de sobra conocido por todas las Comisiones Nacionales de todas las especialidades sanitarias, los colegios profesionales correspondientes, asociaciones científicas en general y por la AEPCP.

Tras el oportuno debate en la reunion antes mencionada de la CNEPC del día 5 del pasado mes de marzo y en relación al contenido planteado en dicha carta, la CNEPC, por unanimidad, decide remitir un escrito a ambos Ministerios expresando la disposición de todos los miembros a su propia renovación y la de la Comisión.

Así mismo en dicho escrito se expresa el profundo malestar por las descalificaciones dirigidas a la CNEPC y su presidenta en particular, que se valoran como falsas y sin ningún fundamento, más cuando en todas y cada una de las decisiones adoptadas el representante del COP ha mostrado su total acuerdo.

La AEPCP, al igual que otras importantes asociaciones y colectivos profesionales y académicos de la Psicología en general y de la Psicología Clínica en particular, así como algunos colegios de psi- cólogos autonómicos y profesionales relevantes de la Psicología Clinica, ha sostenido en relación a todo este asunto su posición de respaldo a los trabajos de la CNEPC y de su representación en la misma, de lo que pormenorizadamente viene dando cuenta en la web y próximamente lo dará en la Revista. Respaldo que los miembros de la CNEPC y yo misma tenemos ocasión ahora de agradecer a todos por su firmeza, celeridad y transparencia, que dan cuenta del acogedor respaldo producido.

En cualquier caso, en lo que concierne a la CNEPC, este plazo de 4 años terminó el pasado mes de noviembre de 2003 y estamos a la espera de la renovación de la misma. Mientras tanto, la CNEPC solicita asesoramiento jurídico a la Administración acerca de la situación legal en que se encuentra, así como del valor de sus decisiones. Según informa expresamente D. Ramón Jiménez, asesor del M. ${ }^{9}$ de Sanidad, y como consta en el acta del 2 del pasado mes de abril, la capacidad legal de la CNEPC es estrictamente plena $y$, hasta tanto se decida la renovación, que señala como proceso prolongado habitualmente, la situación es como en muchas otras CNs de estar «en funciones», y ello a fin de no demorar ni paralizar los trabajos encomendados. En lo que se refiere a la CNEPC particularmente en este momento, para no paralizar la revisión de expedientes de homologación de títulos de especialista con los consiguientes perjuicios para los actualmente cerca de 10.500 solicitantes.

Así que continuamos trabajando cada 15/20 días las reuniones de la CNEPC dando con ello cumplimiento al compromiso contraído de trabajar por la especialidad, tarea que, como es la norma para todas las comisiones nacionales de todas las especialidades, no tiene emolumentos o gratificación económica.

Para finalizar este punto, señalaré que la continuidad de la $A E P C P$ como miem- 
bro de la CNEPC en representación de sociedades científicas, posiblemente sea hoy un hecho consolidado que no debe hacer perder de vista el objetivo de mantenerlo, lo que, por otra parte, no deja de responder al trabajo desempeñado por la Asociación, de manera continuada, a lo largo de los años, sus decisivas aportaciones y con especial relevancia durante el presente período de gestión.

10. La CNEPC creada en noviembre de 1999, tenemos establecida la línea de informar y aclarar a la Junta Directiva, a los socios y a cuantos tengan interés, las confusiones o las actuaciones a desarrollar en base a las informaciones que obran en poder de la CNEPC.

Así por ejemplo hicimos el año pasado por el asunto de la interposición de recursos ante falta de respuesta expresa por la Administración en 6 meses a la solicitud de homologación de título de especialista, cuestión ésta de la que se ocupó la Presidenta de AEPCP y la representante de la Asociación en la CNEPC dando traslado a la Junta Directiva y a los socios a través de escrito aclaratorio, de la información que obraba en poder de la CNEPC al respecto en torno a la superación del plazo de 6 meses establecido en la Orden Ministerial 1107/2002 para la obtención de respuesta de los cerca de 10.500 expedientes de solicitud de homologación del título de especialista en Psicología Clínica y la innecesaria interposición de los mismos dada la obligatoriedad de respuesta de la Administración.

En esta ocasión hay que decir que nos ha planteado la Administración como plazo estimado al menos tres años de trabajo para finalizar la revisión de los actuales expedientes ( $\sin$ entrar en la consideración del aumento que supondrá la llegada de nuevas solicitudes con la publicación del próximo y nuevo Real Decreto de apertura de plazos). Plazo aproximado siempre que se mantuviera el vertiginoso ritmo de trabajo actual, atendiendo para su establecimiento tanto a experiencias anteriores similares y recientes en la Admón. respecto a otras especialidades.

Entiéndase bien: este plazo no es porque la CNEPC así lo quisiera, sino porque junto al alto número de solicitudes y la existencia de cuatro agrupaciones de expedientes por las cuatro disposiciones transitorias con sus respectivos requisitos diferenciados, el circuito de revisión de todos los expedientes implica que son examinados en primer lugar por los funcionarios del Ministerio de Educación, quienes ya pueden tomar decisiones ( $y$ de hecho lo hacen) de denegación, de solicitud de información complementaria o, revisado, dar paso y remitirlos a la CNEPC que, a su vez, ha de valorarlos e informarlos. A continuación, revisa el M. ${ }^{2}$ de Sanidad y entonces se remite de nuevo a M. ${ }^{9}$ Educación. Precisamente la cuestión de los tiempos para alcanzar la finalización del proceso, fue uno de los argumentos para buscar y aceptar fórmulas que lo agilizasen, sin descuidar la calidad necesaria para llevarlo a cabo. $Y$ entre esas fórmulas, estuvo como ya he dicho antes, la revisión de los expedientes por disposiciones transitorias, y no atendiendo exclusivamente a criterios de estricto orden de llegada y registro de la Administración.

Otra fórmula fue reclamar un aumento del número de personas que, en el Ministerio de Educación, estuvieran dedicadas a la revisión previa de expedientes, lo que se ha hecho. $Y$ he de decir que su trabajo es impecable y es facilitador de la tarea de la CNEPC.

Tengo el convencimiento de que en relación a la especialidad hemos alcanzado logros importantes en cuanto a su institucionalización, su ubicación en el Sistema Nacional de Salud, los inicios de la configuración de un colectivo de per- 
fil identificado/ble de psicólogos especialistas sanitarios, su Programa de Formación -más aún si valoramos la posibilidad de la aprobación del nuevo Programa-, las rotaciones, los requisitos para la acreditación de servicios sanitarios como Unidades Docentes Acreditadas (UDAs), el sistema de auditorías, la existencia de un número de UDAs en el Estado que parece suponer que este inicio cuenta con suficiente base para los imprescindibles avances en su incremento, etc., pero no hay que olvidar la juventud y vulnerabilidad de la especialidad. Confío en la capacidad de reconducción de los procesos, de la elaboración, de la maduración, y estoy convencida de que somos y seremos capaces de hacerlo también ahora.

Por todo esto precisamos defender activamente la especialidad:

- Su calidad en la formación.

- Su calidad en los procesos de homologación de títulos de especialista.

- Su ubicación en el Sistema Nacional de Salud (reclasificación de plazas y ampliación de presencia en servicios del SNS).
- El seguimiento de la nueva normativa que desarrollará las nuevas leyes mencionadas y modificación de aspectos antes señalados en las mismas.

- El desarrollo y aumento de plazas PIR o de formación de especialistas en Psicología Clínica.

- El desarrollo y aumento de plazas de psicólogos clínicos en el SNS que permita: A) la actualización imprescindible de las desfasadas ratios existentes de número de habitantes/especialista en Psicología Clínica, que no se han visto modificadas desde comienzos de los años noventa, mientras el aumento de demanda de atención de Psicología Clínica en los servicios ha sido exponencial. Aumento de demanda que a su vez da cuenta y ratifica el acertado camino desarrollado por la especialidad.

B) contar con un número más adecuado de psicólogos clínicos en las UDAs.

En Madrid, a 30 de junio de 2004 